\title{
Results from the EDGES Survey
}

\section{Liese van Zee and the EDGES Team}

Department of Astronomy, Indiana University, Bloomington, IN 47405 email: Ivanzee@indiana.edu

\begin{abstract}
Results are presented from a deep imaging survey with the Spitzer Space Telescope which was designed to identify and measure the faint stellar populations around nearby galaxies. The Extended Disk Galaxy Exploration Science (EDGES) Survey includes a sample of 92 nearby galaxies with a range of morphological types and environments. The observations include a field-of-view of at least 5 times the optical size and are deep enough to detect stellar mass surface densities of several hundredths of a solar mass per square parsec. The observations reveal extended stellar features, such as stellar disks and stellar streams, around many of the target galaxies, as expected from hierarchical galaxy formation scenarios.
\end{abstract}

Keywords. galaxies: evolution, galaxies: halos, galaxies: spiral, galaxies: structure

\section{Introduction}

Deep imaging observations provide unique opportunities to trace the baryonic content of the universe to low surface densities in the outer regions of galaxies. For example, recent deep optical imaging observations have revealed faint stellar distributions that extend factors of 2-3 beyond the bright component in nearby galaxies (e.g., M31, Ibata et al. 2007; NGC 5907, Martínez-Delgado et al. 2008; NGC 4013, Martínez-Delgado et al. 2009). These recent discoveries motivate an increased need to understand galaxy formation and the growth of galaxy disks in the context of the wide range of galaxy properties observed today. We thus undertook the Extended Disk Galaxy Exploration Science (EDGES) Survey with the Spitzer Space Telescope to obtain deep wide-field near-infrared observations of a large sample of nearby galaxies. These observations are designed to trace the full extent of the stellar component in a statistical sample of normal galaxies. Indeed, since galaxies appear to be much larger entities than one might expect based only on their high surface brightness components, the EDGES Survey is designed to have both sufficient field-of-view and depth to trace the diffuse light associated with nearby galaxies in order to understand the full distribution of baryons in nearby systems.

\section{The EDGES Survey}

In order to explore the relationship between the extended stellar distribution and global properties, the well-defined EDGES sample includes nearby galaxies with a diverse range of morphological type, luminosity, inclination angle, and environment. The selected galaxies were drawn from a parent sample that included all galaxies listed in NED $\dagger$ with velocities less than $3000 \mathrm{~km} \mathrm{~s}^{-1}$ and $|\mathrm{b}|>20^{\circ}$. The selection criteria included both a minimum and maximum distance cut $(2 \mathrm{Mpc}<\mathrm{d}<15 \mathrm{Mpc})$, a minimum and maximum angular size cut $\left(2^{\prime}<D_{25}<13^{\prime}\right)$, an apparent magnitude limit $\left(\mathrm{m}_{B}<16\right)$, and a very strict galactic latitude constraint $\left(|\mathrm{b}|>60^{\circ}\right)$. The latter criterion was imposed based on

$\dagger$ NASA/IPAC Extragalactic Database, which is operated by the Jet Propulsion Laboratory, California Institute of Technology, under contract with the National Aeronautics and Space Administration. 
experience with data from two pilot projects in Spitzer Cycle 6, where we discovered that even moderate galactic latitude fields have significant contamination by foreground stars at the depths of these images (e.g., Barnes et al. 2014). In addition, to minimize confusion with background structure, we excluded all potential targets within $20^{\circ}$ of the center of the Virgo cluster. Because the combination of a distance cut and an apparent magnitude limit resulted in a nearly complete parent sample for galaxies brighter than $\mathrm{M}_{B}$ of -15 , the sample was further limited to those galaxies brighter than $\mathrm{M}_{B}$ of -14 in order to maintain a nearly complete parent sample while still including a representative sample of low mass galaxies. Finally, fields that would require masking a significant fraction of the field-of-view due to either foreground (stars) or background (galaxy clusters) source contamination were excluded. This combination of selection criteria ensures that the EDGES Survey includes an unbiased sample of galaxies that are representative of systems that encompass the majority of baryonic mass in this volume element.

The 92 galaxies in the EDGES sample were observed with the Spitzer Space Telescope during 2011-2013 (Cycle 8) for a total integration time of 1800s per position on the sky at both $3.6 \mu \mathrm{m}$ and $4.5 \mu \mathrm{m}$. The Spitzer observations were processed with standard MOPEX mosaicking software and include distortion corrections, rotation of the individual frames, bias structure and bias drift corrections, and image resampling to 0'.75 pixels using drizzling techniques (see Staudaher et al. 2015). It is important to note that the EDGES observations are significantly deeper and cover a larger area than most other Spitzer observations of nearby galaxies (e.g., SINGS, Kennicutt et al. 2003, LVL, Dale et al. 2009, and $\mathrm{S}^{4} \mathrm{G}$, Sheth et al. 2010) which have typical integration times of only $240 \mathrm{~s}$ and fields-of-view that are often only slightly larger than $R_{25}$.

\section{Preliminary Results from the EDGES Survey}

As expected, the deep, wide field-of-view Spitzer $3.6 \mu \mathrm{m}$ and $4.5 \mu \mathrm{m}$ images reveal the extent and frequency of faint stellar populations associated with normal galaxies. The low surface density stellar structures identified in the EDGES survey include distinct features, such as stellar streams and tidal tails, as well as diffuse structures such as extended stellar disks. A handful of galaxies in the EDGES Survey were known previously to have extended stellar features based on deep optical imaging observations; the Spitzer observations confirm these results. However, it is also important to note that the Spitzer observations have the advantage that near-infrared data are less sensitive to star formation history and dust extinction; thus, near-infrared surface brightness can be converted more directly to stellar mass surface density than comparable optical data.

The majority of the dramatic stellar structures identified in the EDGES survey are associated with galaxies that are interacting. Galaxy interactions and mergers are transformational events that are crucial to the formation and evolution of galaxies, can induce starburst activity, and are the cornerstone of hierarchical models of galaxy formation. Even interactions with low mass companions can result in extended stellar structure. For example, EDGES observations of NGC 5005 reveal both a diffuse extended stellar disk and a stellar stream that connects from the primary galaxy to a faint companion to the north (Figure 1). As presented in Richards et al. (2015), we find that the stellar disk extends well beyond the neutral gas distribution in this large galaxy. This result is unusual insofar as many galaxies with extended stellar disks are also known to have extended neutral gas disks (e.g., M83, Thilker et al. 2005). It is possible that the diffuse stellar disk associated with NGC 5005 is representative of the future fate of galaxies like M83 that currently have both extended gaseous disks and extended star formation activity. 

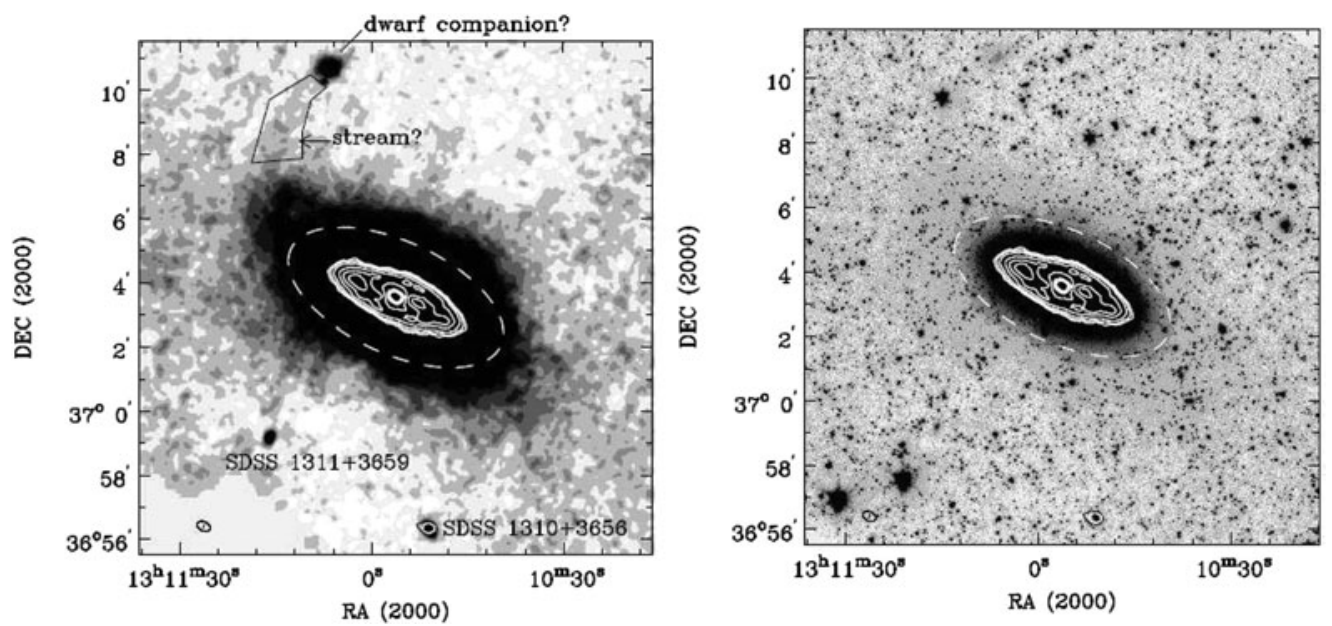

Figure 1. Spitzer $3.6 \mu \mathrm{m}$ images of NGC 5005 reveal an extended stellar distribution and a faint stream to the northeast. The editted and smoothed image on the left illustrates the extent of the stellar distribution that can be detected in the deep EDGES observations. The white ellipse indicates the break radius for the stellar surface brightness profile; the white contours indicate the extent of the neutral hydrogen disk. A diffuse stellar stream may connect NGC 5005 with a low mass companion to the northeast. The intrinsicaly faint nature of the stream and potential companion galaxy are evident in the image on the right. Adapted from Richards et al. (2015).

\section{Conclusions}

The EDGES Survey was designed to detect and measure the extended stellar features associated with the growth and evolution of galaxies in a $\Lambda$ CDM universe. The deep, wide field-of-view observations of 92 nearby galaxies at $3.6 \mu \mathrm{m}$ and $4.5 \mu \mathrm{m}$ reveal extended stellar structures associated with many galaxies, including stellar streams and diffuse light. The near-infrared observations are sensitive to extended structures regardless of star formation history, and thus provide the most complete census and quantitative measures of the physical properties of low surface brightness features identified around nearby galaxies.

\section{Acknowledgements}

This work is based on observations made with the Spitzer Space Telescope, which is operated by the Jet Propulsion Laboratory, California Institute of Technology under a contract with NASA. Support for this work was provided by NASA through an award issued by JPL/Caltech.

\section{References}

Barnes, K. L., van Zee, L. et al. 2014, ApJ, 789, 126

Dale, D. A. et al. 2009, ApJ, 703, 517

Ibata, R., et al. 2007, ApJ,671, 1591

Kennicutt, R. C., et al. 2003, PASP, 115, 928

Martínez-Delgado, D., et al. 2008, ApJ, 689, 184

Martínez-Delgado, D., et al. 2009, ApJ, 692, 955

Richards, E. E., van Zee, L. et al. 2015, MNRAS, 449, 3981

Sheth, K. et al. 2010, PASP, 122, 1397

Staudaher, S. M. et al. 2015, MNRAS, 454, 3613

Thilker, D. A., et al. 2005, ApJ, 619, L79 